\title{
Automatic Construction of Statistical Shape Models for Vertebrae
}

\author{
Meike Becker, Matthias Kirschner, Simon Fuhrmann, and Stefan Wesarg \\ GRIS, TU Darmstadt, Fraunhoferstraße 5, 64287 Darmstadt, Germany \\ meike.becker@gris.tu-darmstadt.de
}

\begin{abstract}
For segmenting complex structures like vertebrae, a priori knowledge by means of statistical shape models (SSMs) is often incorporated. One of the main challenges using SSMs is the solution of the correspondence problem. In this work we present a generic automated approach for solving the correspondence problem for vertebrae. We determine two closed loops on a reference shape and propagate them consistently to the remaining shapes of the training set. Then every shape is cut along these loops and parameterized to a rectangle. There, we optimize a novel combined energy to establish the correspondences and to reduce the unavoidable area and angle distortion. Finally, we present an adaptive resampling method to achieve a good shape representation. A qualitative and quantitative evaluation shows that using our method we can generate SSMs of higher quality than the ICP approach.
\end{abstract}

\section{Introduction}

Segmentation of vertebrae is necessary for several clinical applications such as the treatment of severe herniated vertebral disks or the insertion of pedicle screws. Segmenting vertebrae is a challenging task 12 . Besides local image artifacts or noise, different vertebrae lie close together and are difficult to distinguish from each other and from the adjacent rib cage. Therefore, a general approach is to use a-priori information by means of statistical shape models (SSMs) to make the segmentation more robust. An SSM contains information about the mean of the training set and the possible variations from the mean. For a detailed discussion of SSMs we refer to the review of Heimann et al. [10].

One of the main challenges using SSMs is to establish correspondences in the training data set. Each training shape has to be represented by the same number of 3D points (also called landmarks) and landmarks representing the same anatomical feature should have the same index. A common way to solve this problem is to parameterize every surface to a common base domain and to establish the correspondences on this parameter space. Current work in this context focuses on genus 0 objects such as liver or kidney 25914. Visually spoken, the genus describes the number of holes of a surface. Only Lamecker et al. [13] presented a method independent of the genus, where they employ manually defined patches for each shape and parameterize every patch to a disk. Using this approach, discontinuities may occur along the cuts. 
Reference Mesh

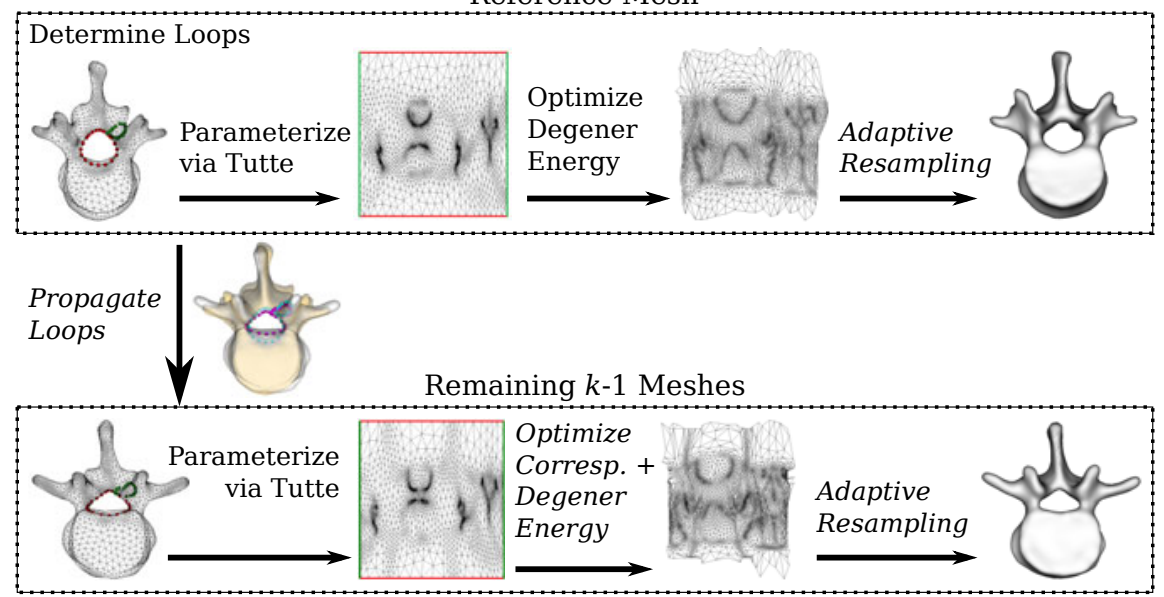

Fig. 1. Overview of our algorithm. Our contributions are printed in italics.

In this work we present an automated approach designed for closed surfaces of genus 1 like vertebrae (see Figure 1). We do not rely on a simple IterativeClosest-Point (ICP) based approach [1] only, since this algorithm does not take into account the triangle structure of the mesh. Hence, flipped triangles in the aligned shapes may occur (see Figure 2).

Related work: Concerning the establishment of correspondences on the parameter space, approaches similar to ours are those of Brett and Taylor [2] and Meier and Fisher [14. Both work on a parameter space but do not reduce any distortion. Another approach is the work of Davies et al. [5], which is based on information theory. Here, the so-called Minimum Description Length (MDL) function is minimized over the parameter space. We do not use this objective function, since it is more costly than our approach.

A lot of research has been done in the area of mesh parameterization. For a detailed overview we refer to the Siggraph Course Notes of Hormann et al. [1]. When mapping a 3D shape to a plane rectangle, distortion of angle and area is unavoidable in most cases. Tewari et al. [17] presented an algorithm for meshing genus-1 point clouds via parameterization. Since they do not sample on the parameter space but reconstruct the shape by inverting the parameterization, they do not have to consider distortion. Tailored to the parameterization of genus-1 meshes is the work of Steiner and Fischer [16. While they consider angular distortion, no area distortion is taken into account. However, for a highquality resampling on the parameter space, it is crucial to minimize both angular and area distortion [6 11]. Only few algorithms exist which minimize both kinds of distortion simultaneously. We choose Degener et al.'s approach [6] since they use a differentiable energy and can obtain a parameterization which is optimal for uniform sampling. 

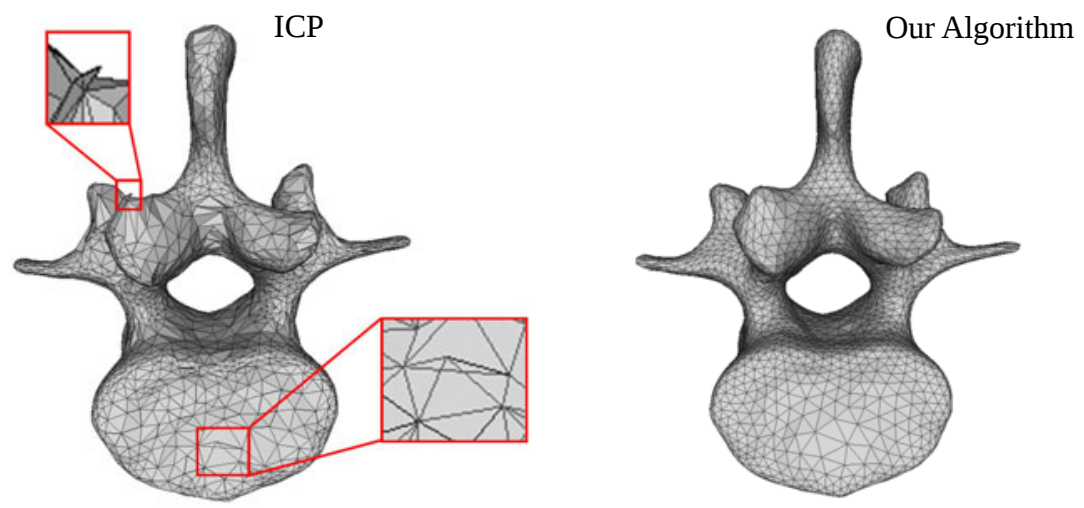

Fig. 2. Comparison of vertebrae after correspondence establishment (with the same number of triangles). Using ICP (left), triangle flips occur (red boxes), while our algorithm prevents them (right).

Contributions: Our work has the following contributions:

1. We automatically construct a statistical shape model for vertebrae. To the best of our knowledge, this is the first approach without any manual interaction apart from the simple ICP approach.

2. We present an algorithm for the consistent propagation of two reference loops to the remaining training set.

3. We introduce a correspondence term based on point-to-point distances and combine it with Degener's energy [6] in order to establish both accurate correspondences and a good reconstruction quality after uniform sampling.

4. We present a new algorithm for curvature adaptive resampling of the parameter space to further improve the shape representation.

\section{Methods}

The input of our algorithm is a set $S=\left\{s_{i}: i=1, \ldots, k\right\}$ of training surfaces, the so-called shapes, extracted from expert segmented image data. Each shape is approximated by a triangle mesh $\mathcal{M}=(\mathcal{P}, \mathcal{T})$, where $\mathcal{P}$ consists of the approximating points $P_{j}, j=1, \ldots, n$ and $\mathcal{T}$ contains the triangles describing the connectivity of the points. For a given index $j$ of a node we write $P_{j}$ for the 3D world space coordinates and $p_{j}$ for the $2 \mathrm{D}$ parameter space coordinates. In the following we describe how to establish the correspondences for this training set.

Reference Mesh: We select a randomly chosen reference shape $s_{\text {Ref }}$. From topology we know that we have to cut a surface of genus 1 along two cut loops in order to make it homeomorphic to a rectangle in the plane. For an introduction to topology we refer to the book of Munkres [15]. Let $G_{\text {Ref }}=\left(V_{\operatorname{Ref}}, K_{\mathrm{Ref}}\right)$ be the corresponding graph to $s_{\text {Ref }}$ with vertices $V_{\text {Ref }}$ and edges $K_{\text {Ref }}$. On this 
graph, we determine the shortest set of loops with a common basepoint using the automatic algorithm of Erickson and Whittlesey [7. We do this for every point of the triangle mesh and choose the shortest set of loops over all basepoints. Here, we use the Euclidean distance to weight the edges. The found loops span the fundamental group [15] of the vertebra given the basepoint $b_{\text {Ref }}$.

After the two cut loops have been found on the reference mesh, we parameterize it to a rectangle using Tutte's graph embedding method [18] with uniform weights. For mapping the boundary, we map each loop to an edge of the rectangle and create a copy which we map to the opposite edge (see Figure 1). In doing so, opposite edges are identified, so that the corresponding quotient space [15] of the rectangle is homeomorphic to a torus.

The distortion of angle and area on the parameter space leads to a bad shape representation when sampling uniformly on the parameter space. Degener et al. 6] minimize in every node an energy which reduces both angle and area distortion. It is defined as $E_{j}^{\text {Deg }}=\sum_{T \in 1 \text {-ring }(j)} E_{\text {angle }}(T) E_{\text {area }}(T)$ for every node $j=1, \ldots, n$, where the set $1-\operatorname{ring}(j)$ contains all triangles $T$ such that $j \in T$,

$$
\begin{aligned}
& E_{\text {angle }}(T)=\frac{a^{2} \cdot \cot \alpha+b^{2} \cdot \cot \beta+c^{2} \cdot \cot \gamma}{2 \operatorname{area}(T)} \text { and } \\
& E_{\text {area }}(T)=\frac{\operatorname{area}\left(T_{3 D}\right)}{\operatorname{area}(T)}+\frac{\operatorname{area}(T)}{\operatorname{area}\left(T_{3 D}\right)} .
\end{aligned}
$$

The variables $a, b$ and $c$ represent the edge lengths of the triangle $T$ in the parameter space and $\alpha, \beta$ and $\gamma$ describe the opposite interior angles of the corresponding triangle $T_{3 D}$ in the world space. We use this differentiable energy to reduce the area and angle distortion on the parameter space. We additionally reduce the distortion at the boundary by employing a free-boundary optimization that allows the boundary to move. Points leaving the rectangle on one side, are inserted again on the opposite, identified side.

Remaining training set: Since we want to cut approximately along the same anatomical paths for each training shape, i. e. find consistent loops, we propagate the two reference loops to the remaining training shapes as follows: We first align every remaining training shape $s_{i}, i=2, \ldots, k$, to the reference mesh using the Iterative-Closest-Point (ICP) method, integrating normals in the similarity criterion. We then propagate the reference basepoint $b_{\text {Ref }}$ by choosing the nearest neighbor on $s_{i}$. Again, we apply Erickson and Whittlesey's algorithm to the propagated basepoint $b_{i}$, but we introduce a new cost function for the edges based on the distance to the reference loops. Let $L^{\text {Ref }}$ contain the points of the two reference loops. Then the cost function $c_{i}: K_{i} \longrightarrow \mathbb{R}_{+}$for the graph $G_{i}=\left(V_{i}, K_{i}\right)$ of the shape $s_{i}$ is defined as follows:

$$
c_{i}\left(j_{1}, j_{2}\right)=0.5\left[\min _{\zeta \in L^{\operatorname{Ref}}}\left(\left\|P_{j_{1}}^{i}-P_{\zeta}^{\mathrm{Ref}}\right\|\right)+\min _{\xi \in L^{\operatorname{Ref}}}\left(\left\|P_{j_{2}}^{i}-P_{\xi}^{\mathrm{Ref}}\right\|\right)\right] .
$$

In this way we ensure that the propagated loop $L^{i}$ is close to the reference loop $L^{\text {Ref }}$. Furthermore, Erickson and Whittlesey's algorithm guarantees that 
we obtain a shape homeomorphic to a rectangle by cutting along the propagated loops. Hence, the propagation of the loops is assured to be successful.

Subsequently, the current shape $s_{i}$ is parameterized in the same way as the reference mesh but with the difference that we also establish correspondences: Remember that every shape $s_{i}$ is aligned to the reference mesh via ICP. This global alignment for establishing correspondences can lead to flipped triangles as the ICP algorithm ignores the triangulation. Therefore, we minimize an energy over the parameter space. This energy is defined as $E_{j}=E_{j}^{\text {Cor }}+\lambda E_{j}^{\text {Deg }}$, where $E_{j}^{\text {Cor }}$ describes the correspondence term, $E_{j}^{\text {Deg }}$ is responsible for reducing the distortion and $\lambda \in \mathbb{R}_{0}^{+}$is a free parameter which has to be chosen appropriately. Let $N_{\mathrm{ICP}}^{q}(j)$ contain the indices of the $q$ nearest neighbors (in our case $q=8$ ) on the reference shape of the node $j$ on the current shape $s_{i}$. For the correspondence term we use a soft-nearest neighbor approach and define our energy as

$$
E_{j}^{\text {Cor }}=\sum_{k \in N_{\mathrm{ICP}}^{q}(j)} w_{j k}\left\|p_{j}^{i}-p_{k}^{\mathrm{Ref}}\right\|,
$$

where the weights $w_{j k}=\exp \left(-\left\|P_{j}^{i}-P_{k}^{\text {Ref }}\right\|\right)$ describe the distance between the point $j$ on $s_{i}$ and the point $k$ on $s_{\text {Ref }}$ in the 3D world space. To achieve a good shape representation, we add to this correspondence term the distortion energy $E_{j}^{\text {Deg }}$ which we already used for $s_{\text {Ref }}$. Like Degener et al. [6], we minimize the combined energy $E_{j}$ for $j=1, \ldots, n$ using the Polak Ribière method. Triangle flips are avoided by restricting every node to the kernel of its 1-ring.

In this way we parameterize every shape consistently to the parameter space, where we establish correspondences and reduce the resulting distortion.

Adaptive Resampling: Once the parameterizations are in correspondence, we can define a common sampling grid in order to reconstruct corresponding landmarks for each shape from its parameterization. During reconstruction, a compromise between two conflicting goals must be established: The more landmarks we use, the greater becomes the gap between the number of training shapes and their dimension. Conversely, we need enough landmarks to obtain an accurate model of the shape which contains every relevant feature. Obtaining a sparse, yet accurate description of the vertebral shape is particularly challenging. While the vertebral body has a relatively simple geometry, the vertebral processes are thin regions with high curvature. Therefore, it is reasonable to use a curvature adaptive strategy for landmark sampling. Adaptive sampling schemes for SSM construction have been proposed by Heimann et al. 9] and Cates et al. 3]. Heimann et al. use adaptive sampling exclusively to compensate for area distortion in the parameterizations. The particle system method of Cates et al. adaptively oversamples features with high curvature, but does not inherently produce a consistent triangulation for all shapes.

We first construct a dense, uniform sampling grid that is used to sample the parameter space. The large number of sampling points and the low distortion of the parameter spaces ensure that all details of the input shapes are preserved. We average all densely reconstructed shapes to a mean mesh $\overline{\mathcal{M}}$, and use a 
state-of-the-art remeshing algorithm 8 in order to obtain a sparse, curvature adaptive representation $\overline{\mathcal{M}}_{\text {Adap }}$ of $\overline{\mathcal{M}}$. Because the initial, uniform sampling grid defines a parameterization of $\overline{\mathcal{M}}$, and all points of $\overline{\mathcal{M}}_{\text {Adap }}$ lie on the surface of $\overline{\mathcal{M}}$, we can compute the parameter space coordinates of the points of $\overline{\mathcal{M}}_{\text {Adap }}$ by simple linear interpolation. These parameter space coordinates define the final, adaptive sampling grid. It is used to sample sparse landmark representations of all input shapes, from which we compute the SSM.

\section{$3 \quad$ Experiments and Results}

For our experiments, we used a training set of 14 manually segmented lumbar vertebrae (L1-L3) from CT scans of five different patients. We established correspondences for this training set with our algorithm using different $\lambda$. For comparison, we also used the ICP algorithm including normals in the similarity criterion as done by Brett and Taylor 2. Then we constructed the corresponding SSMs using the standard approach by Cootes et al. 4. The different $\lambda$ for our algorithm indicate how much weight we give relatively to the Degener term for the different SSMs. As the Degener term is numerically much higher than the correspondence term, we note that the factor $\lambda$ does not reveal anything about the absolute ratio of the two energies.

In Figure 3 we show that the local artifacts caused by ICP can be avoided with our algorithm, which leads to SSMs with higher quality. Furthermore, we evaluated the SSMs using the measures of generalization and specificity as proposed by Davies et al. [5] (see Figure 4). For calculating those measures, we sampled 1000 normally distributed model instances and compared them to the

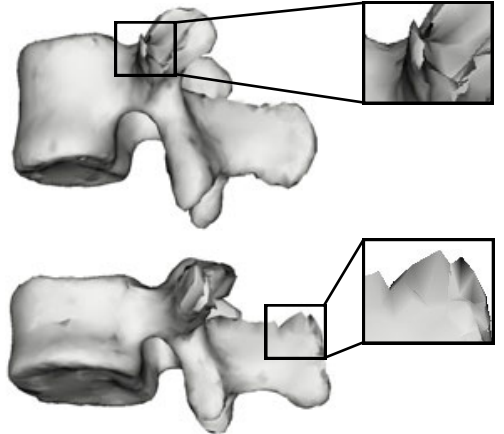

(a) Mode 1 of SSM constructed via ICP using normals

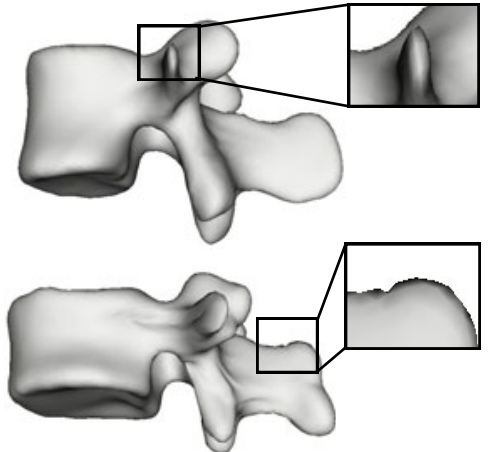

(b) Mode 1 of SSM constructed using our algorithm with $\lambda=0.00025$

Fig. 3. Comparison of SSMs. The first mode of the respective SSM is shown for $2 \sigma$ (first row) and $-2 \sigma$ (second row), where $\sigma$ describes the standard deviation. Local artifacts occur in the SSM constructed via ICP using normals. These artifacts are eliminated with our algorithm, which leads to an SSM of higher quality. 

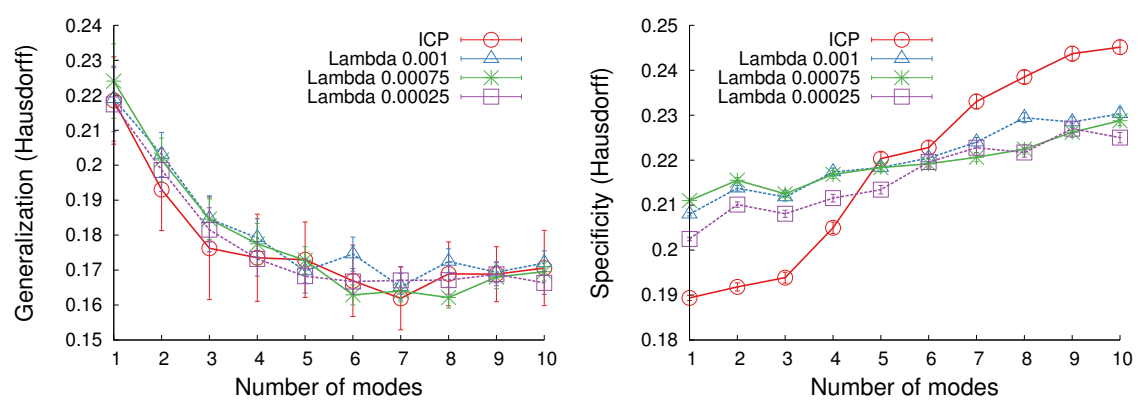

Fig. 4. Evaluation of SSMs using the generalization and specificity measure [5, vertical bars indicating the standard error of the mean. The $x$-axis gives the number of modes used in the experiment. The generalization values (left) are very similar, while our algorithm has better specificity values (right) from mode 5 on.

training set using the Hausdorff metric [15. We did not use the compactness measure since the ICP algorithm may lead to a loss of detail, but loss of detail leads to a better compactness. Hence, we cannot get reliable information about the quality of the SSM using the compactness measure.

We observe that from mode 5 on the specificity measure of our algorithm is better than that of the ICP, while for the generalization, results lie too close together to determine any rank order. To be more specific, all values of our algorithm lie within the interval of the standard error of the ICP.

\section{Discussion}

We presented an approach for automatically solving the correspondence problem for vertebral shapes. To the best of our knowledge it is the first automated approach to construct SSMs for vertebrae aside from the simple ICP approach. We ensure that for every shape of the training set we cut approximately along the same anatomical paths. When every shape is parameterized to the rectangle, we optimize the correspondence by minimizing a novel combined energy function based on point-to-point distances which additionally penalizes distortion.

For a good reconstruction of the shape, we developed an enhanced method for adaptive resampling which is independent of the underlying parameter space. In contrast to the method of Heimann et al. 9], we do not need to use different maps to sample different regions of the parameter space, even if the parameter space is the unit sphere. The remeshing algorithm does not only distribute landmarks adaptively according to curvature, but also favors equiangular triangles. Thus, it implicitly compensates for small distortion in the parameterizations.

The benefit of our approach over ICP is that it is designed to avoid triangle flips and thus ensures a topological consistent shape model. Experiments show that our SSMs generalize as well as the ICP model, while their specificity is even better. Another advantage of our method is its generality in the sense that it can be used for any surface of genus 1 and furthermore, any (differentiable) objective 
function can be used for the correspondence establishment on the parameter space. Hence, it would be interesting to test other correspondence energies such as the MDL function [5] to see if the correspondences can be further improved.

\section{References}

1. Besl, P.J., Mckay, H.D.: A method for registration of 3-D shapes. IEEE Trans. Pattern Anal. Mach. Intell. 14(2), 239-256 (1992)

2. Brett, A., Taylor, C.: Construction of 3D shape models of femoral articular cartilage using harmonic maps. In: Delp, S., DiGoia, A., Jaramaz, B. (eds.) MICCAI 2000. LNCS, vol. 1935, pp. 1205-1214. Springer, Heidelberg (2000)

3. Cates, J.E., Fletcher, P.T., Styner, M.A., Shenton, M.E., Whitaker, R.T.: Shape modeling and analysis with entropy-based particle systems. In: Karssemeijer, N., Lelieveldt, B. (eds.) IPMI 2007. LNCS, vol. 4584, pp. 333-345. Springer, Heidelberg (2007)

4. Cootes, T.F., Taylor, C.J., Cooper, D.H., Graham, J.: Active shape models their training and application. Comput. Vis. Image Underst. 61, 38-59 (1995)

5. Davies, R.H., Twining, C.J., Taylor, C.J.: Statistical Models of Shape: Optimisation and Evaluation. Springer, London (2008)

6. Degener, P., Meseth, J., Klein, R.: An adaptable surface parameterization method. In: 12th International Meshing Roundtable 2003, Santa Fe, pp. 201-213 (2003)

7. Erickson, J., Whittlesey, K.: Greedy optimal homotopy and homology generators. In: 16th ACM-SIAM Symposium on Discrete Algorithms, pp. 1038-1046. SIAM, Vancouver (2005)

8. Fuhrmann, S., Ackermann, J., Kalbe, T., Goesele, M.: Direct resampling for isotropic surface remeshing. In: Vision, Modeling, and Visualization 2010, pp. 9-16. Eurographics Association, Siegen (2010)

9. Heimann, T., Wolf, I., Meinzer, H.P.: Automatic generation of 3D statistical shape models with optimal landmark distribution. Methods Inf. Med. 46(3), 275-281 (2007)

10. Heimann, T., Wolf, I., Meinzer, H.P.: Statistical shape models for 3D medical image segmentation: A review. Medical Image Analysis 13(4), 543-563 (2009)

11. Hormann, K., Lévy, B., Sheffer, A.: Mesh parameterization: Theory and practice. In: SIGGRAPH 2007 Course Notes, vol. 2. ACM Press, San Diego (2007)

12. Klinder, T., Ostermann, J., Ehm, M., Franz, A., Kneser, R., Lorenz, C.: Automated model-based vertebra detection, identification, and segmentation in CT images. Medical Image Analysis 13(3), 471-482 (2009)

13. Lamecker, H., Lange, T., Seebass, M.: A statistical shape model for the liver. In: Dohi, T., Kikinis, R. (eds.) MICCAI 2002. LNCS, vol. 2489, pp. 421-427. Springer, Heidelberg (2002)

14. Meier, D., Fisher, E.: Parameter space warping: shape-based correspondence between morphologically different objects. IEEE Trans. Med. Imaging 21(1), 31-47 (2002)

15. Munkres, J.R.: Topology. Prentice Hall, Upper Saddle River (2000)

16. Steiner, D., Fischer, A.: Planar parameterization for closed manifold genus-g meshes using any type of positive weights. J. Comput. Inf. Sci. Eng. 5, 118-125 (2005)

17. Tewari, G., Gotsman, C., Gortler, S.J.: Meshing genus-1 point clouds using discrete one-forms. Computers and Graphics 30(6), 917-926 (2006)

18. Tutte, W.T.: How to draw a graph. London Math. Soc. 13, 743-768 (1963) 